\title{
Optimal algorithms for computing multiple integrals
}

\author{
Bandyrskii B. ${ }^{1}$, Hoshko L. ${ }^{1}$, Lazurchak I. ${ }^{2}$, Melnyk M. ${ }^{2}$ \\ ${ }^{1}$ Lviv Polytechnic National University \\ 12 S. Bandera str., 79013, Lviv, Ukraine \\ ${ }^{2}$ Drohobych Ivan Franko State Pedagogical University \\ 24 Ivan Franko str., UA-82100, Drohobych, Ukraine
}

(Received 15 June 2017)

\begin{abstract}
The article deals with optimization algorithms for implementation of Simpson's cubature rule using the principle of double recalculation in calculating multiple integrals. A comparison is represented for the suggested algorithm with the built-in functions of the application package of computer mathematics by test example of computing integral trigonometric functions. The functionality of the Computing Software Mathematica and Maple use is extended.
\end{abstract}

Keywords: cubature formula, calculations optimization, principle of double recalculation, integral cosine, systems of computer mathematics.

2000 MSC: $60 \mathrm{~J} 10$

UDC: 517.9

\section{Introduction}

Optimization of computing on PC due to a significant reduction of computer costs (computer time of CPU, main memory, number of arithmetical and logical operations, loading of address buses, exchange with external peripherals, etc.) is one of the urgent problems of the modern information society. Analysis of last research and publications has shown that many works [1-3] are dedicated to the theoretical development of classical numerical methods of integration and their modifications. In particular, in [3] the approaches related to the principles of double and multiple recalculating when using the Newton-Cotes quadrature formulas are considered. In the paper [4] there were shown the first attempts to optimize the quadrature formulas, but this approach is not generalized for calculating multiple integrals. The purpose of this article is to solve this problem as well as to develop the optimal scheme of algorithm representation for further software implementation, regardless of the computer platform, including operating systems and software environments.

\section{Description of the model, the subjects, and research methods}

The problem of computing multiple integrals arises in various fields of science. Thus, the application of functional and discrete (FD-) method for solving a boundary value problem in the case of equations of elliptic type [6] needs the calculating the multiple integral over two- or three-dimensional domain $\Omega$, namely:

$$
\stackrel{p}{u}(x)=\int_{\Omega}\{G(x, \xi)+\sum_{n=1}^{p}(-1)^{n} \underbrace{\int_{\Omega} \ldots \int_{\Omega}}_{n} \prod_{i=1}^{n+1} G\left(z^{i-1}, z^{i}\right) \prod_{i=1}^{n} \hat{q}\left(z^{i}\right) d z^{1} \ldots d z^{n}\} f(\xi) d \xi, p=0,1,2, \ldots
$$

where $\stackrel{p}{u}(x)$ is a $p$-rank approximation of the solution, $G(x, \xi)$ is a Green's function of the corresponding differential operator. 
With the help of computer mathematics Mathematica and Maple [5] we are able to find efficiently the bilateral analytical approximations. To assess the norms $\|\widehat{q}\|_{0,2, \Omega}$ and $\|f\|_{0,2, \Omega}$ in Lebesgue space $\mathbf{L}_{2}(\Omega)$, the corresponding double Riemann integrals should be calculated. With this purpose, either the Newton-Cotes cubature formulas or the information and statistical methods Monte Carlo-type ones can be applied. With this, for an error a posteriori estimation the principle of the double recalculation is used, which in its turn at each step requires a 4-times increase of the amount of computing costs. The paper presents an algorithm that optimally (without iterative recalculation at the previously calculated points of the scheme) runs the calculations according to the Simpson's cubature rule, starting with the least possible partition. Thus, in the implementation, the accuracy of the calculated double integral is estimated immediately.

Here is a brief description of the realization of the economic scheme in the case of the Simpson's cubature rule.

To represent the approximate value of the integral on the whole interval it is previously divided into an even number $n=2 m$ of intervals, then for each pair of adjacent intervals a substitution of the integrand is made with the Lagrange interpolation polynomial of the second order (quadratic parabola)

$$
\begin{aligned}
\int_{a}^{b} y(x) d x & =\int_{x_{0}}^{x_{2}} y(x) d x+\ldots+\int_{x_{2 m-2}}^{x_{2 m}} y(x) d x \approx \frac{h}{3}\left(y_{0}+4 y_{1}+y_{2}+\ldots+y_{2 m-2}+4 y_{2 m-1}+y_{2 m}\right) \\
& =\frac{h}{3}\left(\sigma_{0}+4 \sigma_{1}+2 \sigma_{2}\right)
\end{aligned}
$$

where the following designations are agreed

$$
\begin{gathered}
\sigma_{0}=\left(y_{0}+y_{2 m}\right), \quad \sigma_{1}=\sum_{i=1}^{m} y_{2 i-1}, \quad \sigma_{2}=\sum_{i=1}^{m-1} y_{2 i}, \\
y_{i}=y\left(x_{i}\right), \quad x_{i}=a+i h, \quad h=\frac{b-a}{2 m}, \quad i=0, \ldots, 2 m .
\end{gathered}
$$

Since the formulas for $\sigma_{1}$ and $\sigma_{2}$ involve different numbers of internal nodes (odd and even), then the use of a cycle for the simultaneous calculation of these sums requires a separate consideration of the last term in the sum $\sigma_{1}$. The generalized Simpson's method gives the approximation of the order of smallness

$$
R(h)=\underline{O}\left(h^{4}\right) .
$$

Hence it can be concluded that the specified formula is accurate for all integrand functions that represent the algebraic polynomial at least of the third order.

Among the quadrature formulas of higher order, the formula of " $3 / 8$ " can be named. However, its order of accuracy is also the value of the order of smallness $\underline{O}\left(h^{4}\right)$. Further, with the increasing number of nodes $n$ of the interpolation polynomial, the order of accuracy of the Newton-Cotes quadrature formulas is rising in a unit, i.e. the error term is of the order $\underline{O}\left(h^{\left[\frac{2 n+1}{2}\right]}\right)$, that is why nowadays one of the most efficient quadrature formulas constructed on a uniform mesh is the very Simpson's rule [2].

The economic algorithm of calculation by the Simpson's quadrature formula consists in the following. Twice increasing the mesh dimension and using the mentioned formula and the principle of double recalculation, we obtain in the new designations

$$
\begin{gathered}
I_{\mathrm{old}}=\frac{h}{3}\left[\sigma_{0}+4 \sigma_{1}+2 \sigma_{2}\right]=\frac{h}{3}\left[\sigma_{0}+2 \sigma_{1}+2 \sigma_{3}\right], \quad \sigma_{3}=2 \sigma_{1}+2 \sigma_{2}, \\
I_{\text {new }}=\frac{h}{2 \cdot 3}\left(\sigma_{0}+2 \tilde{\sigma}_{1}+2 \tilde{\sigma}_{3}\right), \quad \tilde{\sigma}_{3}=\tilde{\sigma}_{1}+\tilde{\sigma}_{2}, \quad \tilde{\sigma}_{2}=\sigma_{3} .
\end{gathered}
$$

Since the sum of the function values in the nodes with even indices for the new numbering coincides with the sum of all of the function values in the internal nodes of the old mesh, then for the 
implementation of the principle of double recalculation it is sufficient to calculate the function values and their sum only in odd nodes of the new mesh. In the general case, in $k$-iteration $(k=0,1,2, \ldots)$ the calculation formulas take the following forms

$$
\begin{aligned}
I_{k} & =\frac{h_{k}}{3}\left[\sigma_{0}+2 \sigma_{1}^{(k)}+2 \sigma_{3}^{(k)}\right], \quad h_{k}=\frac{b-a}{2^{k+1} n}, \\
\sigma_{0} & =[f(a)+f(b)], \quad \sigma_{1}^{(k)}=\sum_{i=1}^{2^{k} n} f\left(a+(2 i-1) h_{k}\right), \\
\sigma_{2}^{(k)} & =\left\{\begin{array}{ll}
\sum_{i=1}^{n-1} f\left(a+2 i h_{k}\right), & k=0, \\
\sigma_{3}^{(k-1)}, & k>1,
\end{array} \quad \sigma_{3}^{(k)}=\sigma_{1}^{(k)}+\sigma_{2}^{(k)} .\right.
\end{aligned}
$$

Let now consider the two-dimensional space $R^{2}$. If the domain of integration is, for example, a rectangle, then the double integral calculation comes to calculating the iterated integral. In fact, this means the consistent use of quadrature formulas first with respect to one spatial variable $x$, and then with respect to the other $-y$. The order of variables of integration does not matter.

$$
\iint_{D} f(x, y) d x d y=\int_{a}^{b} d x \int_{c}^{d} f(x, y) d y, \quad D=\{(x, y), a \leqslant x \leqslant b, c \leqslant y \leqslant d\} .
$$

If the integration domain $G$ is arbitrary, but it is finite and closed, it can be included as a subdomain into some classic flat figure, the integrand in $G \backslash D$ can be additionally determined by the nul values and the previously described approach can be applied.

Using the partitioning of a rectangular domain $D$ consistently with respect to the variable $x$ and $y$, we obtain the symmetric matrix of the coefficients of the cubature formula

$$
A=\left(\begin{array}{ccccccc}
1 & 4 & 2 & 4 & \ldots & 4 & 1 \\
4 & 16 & 8 & 16 & \ldots & 16 & 4 \\
2 & 8 & 4 & 8 & \ldots & 8 & 2 \\
\ldots & \ldots & \ldots & \ldots & \ldots & \ldots & \ldots \\
4 & 16 & 8 & 16 & \ldots & 16 & 4 \\
1 & 4 & 2 & 4 & \ldots & 4 & 1
\end{array}\right)
$$

Then the double integral calculus can be reduced to the iterated integral and the generalized cubature Simpson's formula takes the form

$$
\iint_{D} f(x, y) d x d y=\sum_{i=0}^{2 n} \sum_{j=0}^{2 m} A_{i j} f\left(x_{i}, y_{j}\right)
$$

where the following designations are agreed

$$
\begin{gathered}
f_{i j}=f\left(x_{i}, y_{j}\right), \quad x_{i}=a+i h_{1}, \quad y_{j}=c+j h_{2}, \\
h_{1}=\frac{b-a}{2 n}, \quad h_{2}=\frac{d-c}{2 m}, \quad i=0, \ldots, 2 n, \quad j=0, \ldots, 2 m .
\end{gathered}
$$

Apply to the Simpson's cubature formulas the principle of double recalculation. Write down Eq. (11) in the form

$$
\begin{aligned}
\iint_{D} f(x, y) d x d y= & \frac{h_{1} h_{2}}{9} \sum_{i=0}^{n-1} \sum_{j=0}^{m-1}\left[\left(f_{2 i, 2 j}+f_{2 i+2,2 j}+f_{2 i+2,2 j+2}+f_{2 i, 2 j+2}\right)\right. \\
& \left.+4\left(f_{2 i+1,2 j}+f_{2 i+2,2 j+1}+f_{2 i+1,2 j+2}++f_{2 i, 2 j+1}\right)+16 f_{2 i+1,2 j+1}\right]
\end{aligned}
$$


Consider these formulas for the initial values $n_{0}=2, m_{0}=2$ and represent them as separate sums

$$
I_{0}=\frac{h_{1}^{(0)} h_{2}^{(0)}}{9}\left[\sigma_{0}^{(0)}+\sigma_{1}^{(0)}+4 \sigma_{2}^{(0)}+16 \sigma_{3}^{(0)}\right]
$$

where

$$
\begin{aligned}
& \sigma_{0}^{(0)}=f_{0,0}^{(0)}+f_{0,4}^{(0)}+f_{4,0}^{(0)}+f_{4.4}^{(0)}, \\
& \sigma_{1}^{(0)}=2\left[f_{0,2}^{(0)}+f_{2,0}^{(0)}+f_{2,4}^{(0)}+f_{4,2}^{(0)}\right]+4 f_{2,2}^{(0)}, \\
& \sigma_{2}^{(0)}=f_{0,1}^{(0)}+f_{1,0}^{(0)}+f_{1,4}^{(0)}+f_{4,1}^{(0)}+f_{0,3}^{(0)}+f_{3,0}^{(0)}+f_{3,4}^{(0)}+f_{4,3}^{(0)}+2\left[f_{1,2}^{(0)}+f_{2,1}^{(0)}+f_{2,3}^{(0)}+f_{3,2}^{(0)}\right], \\
& \sigma_{3}^{(0)}=f_{1,1}^{(0)}+f_{1,3}^{(0)}+f_{3,1}^{(0)}+f_{3,3}^{(0)} .
\end{aligned}
$$

Here the superscript corresponds to the previous numbering of the mesh (grid) (Fig. 1).

Let us further apply the principle of double recalculation, i.e. redouble the values $n=2 n_{0}$ and $m=2 m_{0}$. The formulas (13) take the form

$$
I_{1}=\frac{h_{1}^{(1)} h_{2}^{(1)}}{9}\left[\sigma_{0}^{(1)}+\sigma_{1}^{(1)}+4 \sigma_{2}^{(1)}+16 \sigma_{3}^{(1)}\right],
$$

where

$$
\sigma_{0}^{(1)}=f_{0,0}^{(1)}+f_{0,8}^{(1)}+f_{8,0}^{(1)}+f_{8,8}^{(1)} .
$$

The sums in the nodes with even indices have the forms

$$
\begin{aligned}
\sigma_{1}^{(1)}= & 2\left[f_{0,2}^{(1)}+f_{0,4}^{(1)}+f_{0,6}^{(1)}+f_{2,0}^{(1)}+f_{4,0}^{(1)}+f_{6,0}^{(1)}+f_{2,8}^{(1)}+f_{4,8}^{(1)}+f_{6,8}^{(1)}+f_{8,2}^{(1)}+f_{8,4}^{(1)}+f_{8,6}^{(1)}\right] \\
& +4\left[f_{2,2}^{(1)}+f_{2,4}^{(1)}+f_{2,6}^{(1)}+f_{4,2}^{(1)}+f_{4,4}^{(1)}+f_{4,6}^{(1)}+f_{6,2}^{(1)}+f_{6,4}^{(1)}+f_{6,6}^{(1)}\right] .
\end{aligned}
$$

The sums in the nodes with odd indices have the forms

$$
\begin{aligned}
\sigma_{2}^{(1)}= & f_{0,1}^{(1)}+f_{0,3}^{(1)}+f_{0,5}^{(1)}+f_{0,7}^{(1)}+f_{1,0}^{(1)}+f_{1,8}^{(1)}+f_{3,0}^{(1)}+f_{3,8}^{(1)}+f_{5,0}^{(1)}+f_{5,8}^{(1)} \\
& +f_{7,0}^{(1)}+f_{7,8}^{(1)}+f_{8,1}^{(1)}+f_{8,3}^{(1)}+f_{8,5}^{(1)}+f_{8,7}^{(1)}+2\left[f_{1,2}^{(1)}+f_{1,4}^{(1)}+f_{1,6}^{(1)}+f_{2,1}^{(1)}+f_{2,3}^{(1)}\right. \\
& +f_{2,5}^{(1)}+f_{2,7}^{(1)}+f_{3,2}^{(1)}+f_{3,4}^{(1)}+f_{3,6}^{(1)}+f_{4,1}^{(1)}+f_{4,3}^{(1)}+f_{4,5}^{(1)}+f_{4,7}^{(1)}+f_{5,2}^{(1)}+f_{5,4}^{(1)} \\
& \left.+f_{5,6}^{(1)}+f_{6,1}^{(1)}+f_{6,3}^{(1)}+f_{6,5}^{(1)}+f_{6,7}^{(1)}+f_{7,2}^{(1)}+f_{7,4}^{(1)}+f_{7,6}^{(1)}\right], \\
\sigma_{3}^{(1)}= & f_{1,1}^{(1)}+f_{1,3}^{(1)}+f_{1,5}^{(1)}+f_{1,7}^{(1)}+f_{3,1}^{(1)}+f_{3,3}^{(1)}+f_{3,5}^{(1)}+f_{3,7}^{(1)} \\
& +f_{5,1}^{(1)}+f_{5,3}^{(1)}+f_{5,7}^{(1)}+f_{7,1}^{(1)}+f_{7,3}^{(1)}+f_{7,5}^{(1)}+f_{7,7}^{(1)} .
\end{aligned}
$$

In the formulas (19)-(23) the integration steps will be twice reduced

$$
h_{1}^{(1)}=\frac{h_{1}^{(0)}}{2}=\frac{b-a}{2 n}, \quad h_{2}^{(1)}=\frac{h_{2}^{(0)}}{2}=\frac{d-c}{2 m},
$$

and the points of integration will be calculated by the formulas

$$
x_{i}^{(1)}=a+i h_{1}^{(1)}, \quad y_{j}^{(1)}=c+j h_{2}^{(1)}, \quad i=0, \ldots, 2 n, \quad j=0, \ldots, 2 m .
$$

The optimization calculation scheme for double integrals corresponds to the matrix grid (Fig. 1). 


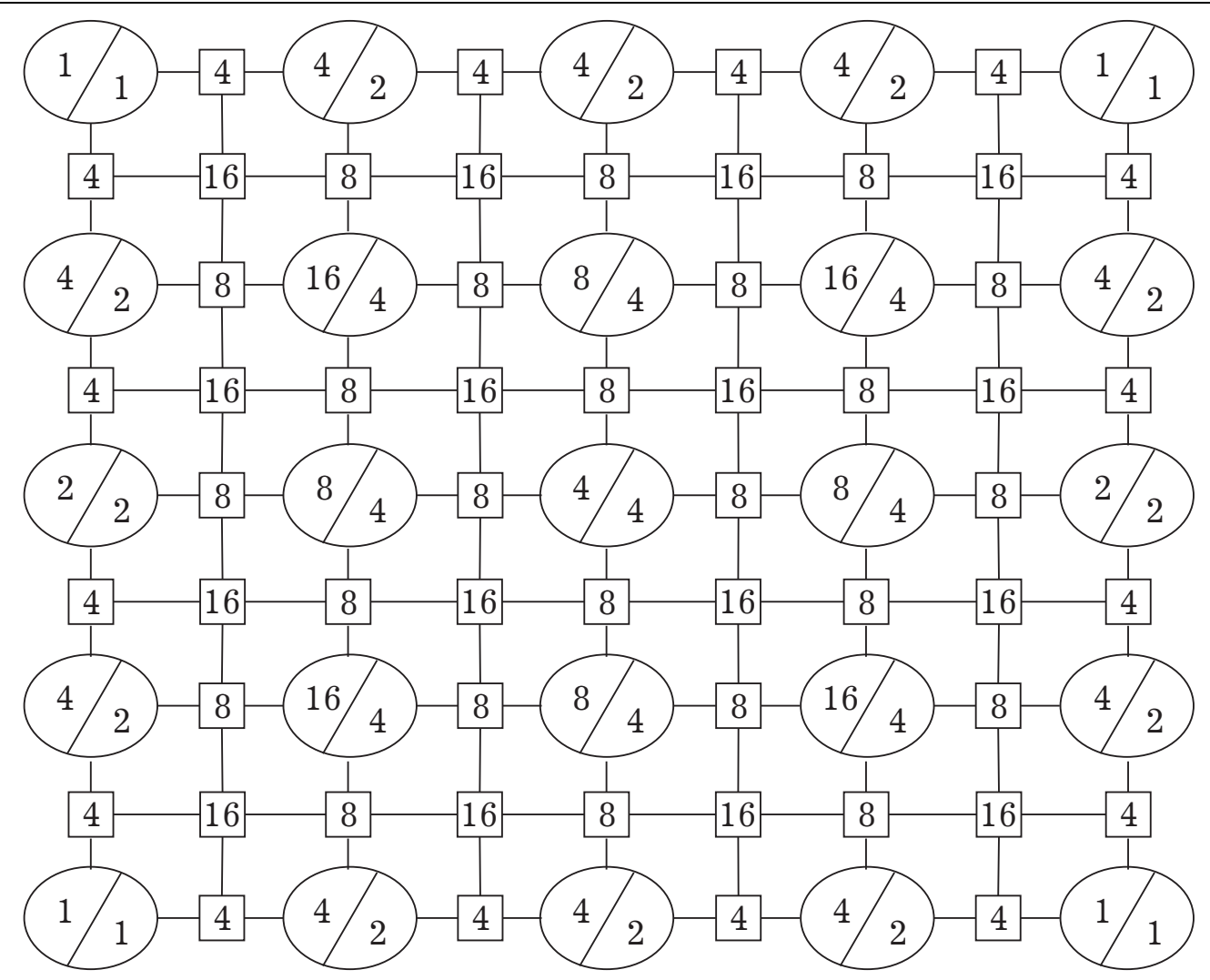

Fig. 1. Matrix grating with coefficients (in circles - for partitioning $n_{0}, m_{0}=2$; in squares - for partitioning $n, m=4)$.

Here the coefficients of the cubature formulas (14)-(18) are represented in circles on the left (top) side and the coefficients for the formulas (19)-(23), which correspond to the double recalculation are presented in the circles on the right (bottom) side and in the squares. You can immediately note that the sums (15) and (20) are equivalent, as they contain the values of the function $f\left(x_{i}, y_{j}\right)$ at the vertices of the rectangular area, i.e.

$$
f_{0,0}^{(0)}=f_{0,0}^{(1)}, \quad f_{0,4}^{(0)}=f_{0,8}^{(1)}, \quad f_{4,0}^{(0)}=f_{8,0}^{(1)}, \quad f_{4,4}^{(0)}=f_{8,8}^{(1)}
$$

Regrouping the terms in (21), we obtain

$$
\begin{aligned}
\sigma_{1}^{(1)}= & \left\{2\left[f_{0,4}^{(1)}+f_{4,0}^{(1)}+f_{4,8}^{(1)}+f_{8,4}^{(1)}\right]+4 f_{4,4}^{(1)}\right\}+2\left\{f_{0,2}^{(1)}+f_{2,8}^{(1)}+f_{2,0}^{(1)}+f_{8,2}^{(1)}+f_{0,6}^{(1)}+f_{6,0}^{(1)}\right. \\
& \left.+f_{6,8}^{(1)}+f_{8,6}^{(1)}+2\left[f_{2,4}^{(1)}+f_{4,2}^{(1)}+f_{4,6}^{(1)}+f_{6,4}^{(1)}\right]\right\}+4\left\{f_{2,2}^{(1)}+f_{2,6}^{(1)}+f_{6,2}^{(1)}+f_{6,6}^{(1)}\right\} .
\end{aligned}
$$

For nodes with even indices in the new numbering, the following correlations have a place

$$
\left.f_{0,4}^{(1)}=f_{0,2}^{(0)}, \quad f_{4,0}^{(1)}=f_{2,0}^{(0)}, \quad f_{4,8}^{(1)}=f_{2,4}^{(0)}, \quad f_{8,4}^{(1)}\right)=f_{4,2}^{(0)}, \quad f_{4,4}^{(1)}=f_{2,2}^{(0)}
$$

then the expression in the first braces (25) fully corresponds to the formula (16). Similarly, due to the truth of the correlations

$$
\begin{array}{lll}
f_{0,2}^{(1)}=f_{0,1}^{(0)}, & f_{2,8}^{(1)}=f_{1,4}^{(0)}, \quad f_{2,0}^{(1)}=f_{1,0}^{(0)}, & f_{8,2}^{(1)}=f_{4,1}^{(0)} \\
f_{0,6}^{(1)}=f_{0,3}^{(0)}, & f_{6,0}^{(1)}=f_{3,0}^{(0)}, \quad f_{6,8}^{(1)}=f_{3,4}^{(0)}, & f_{8,6}^{(1)}=f_{4,3}^{(0)} \\
f_{2,4}^{(1)}=f_{1,2}^{(0)}, & f_{4,2}^{(1)}=f_{2,1}^{(0)}, \quad f_{4,6}^{(1)}=f_{2,3}^{(0)}, & f_{6,4}^{(1)}=f_{3,2}^{(0)}
\end{array}
$$


the expression in the second braces (25) fully corresponds to the formula (17). And finally, the correlations

$$
\begin{aligned}
& f_{2,2}^{(1)}=f_{1,1}^{(0)}, \quad f_{2,6}^{(1)}=f_{1,3}^{(0)}, \\
& f_{6,2}^{(1)}=f_{3,1}^{(0)}, \quad f_{6,6}^{(1)}=f_{3,3}^{(0)}
\end{aligned}
$$

give grounds for comparing the expression in the third braces with the formula (18).

Thus, the next iteration in the double recalculation, there is no need to calculate the value of the sum $\sigma_{1}^{(1)}$ by the formula (25) but immediately determine its value by the recurrent expression

$$
\sigma_{1}^{(1)}=\sigma_{1}^{(0)}+2 \sigma_{2}^{(0)}+4 \sigma_{3}^{(0)} .
$$

This is substantiated by the fact that for the values of the integrand, which correspond to the nodes indicated with circles, there is no need to be calculated. Suffice it to fix their sums according to (26) and reproduce these values with the coefficients written on the right (bottom) side.

The values of the function in new nodes with odd indices, which correspond to the squares in Fig. 1, and accordingly, to the formulas (22), (23) they should be calculated at every step of the double recalculation.

Introduce new designations

$$
\sigma_{4}^{(k)}=\sigma_{1}^{(k)}+2 \sigma_{2}^{(k)}+4 \sigma_{3}^{(k)}, \quad k=0,1,2, \ldots
$$

Then for a couple of formulas (14), (19), we can use the following representation

$$
\begin{gathered}
I_{k}=\frac{h_{1}^{(k)} h_{2}^{(k)}}{9}\left[\sigma_{0}^{(k)}+2 \sigma_{2}^{(k)}+12 \sigma_{3}^{(k)}+\sigma_{4}^{(k)}\right], \\
f_{i, j}^{(k)}=f\left(x_{i}^{(k)}, y_{j}^{(k)}\right), \quad x_{i}^{(k)}=a+i h_{1}^{(k)}, \quad y_{j}^{(k)}=c+j h_{2}^{(k)}, \\
h_{1}^{(k)}=\frac{b-a}{2 n}, \quad h_{2}^{(k)}=\frac{d-c}{2 m}, \quad n=2^{k} n_{0}, \quad m=2^{k} m_{0}, \quad i=0, \ldots, 2 n, \quad j=0, \ldots, 2 m .
\end{gathered}
$$

In general, at the vertices of the rectangle, the integrand values are calculated by the formula

$$
\sigma_{0}^{(k)}=f_{0,0}^{(k)}+f_{0,2 m}^{(k)}+f_{2 n, 0}^{(k)}+f_{2 n, 2 m}^{(k)}
$$

The sums for the values of the indicated function on the surface of the mesh and in the internal nodes will acquire the following form

$$
\sigma_{1}^{(k)}=2 S_{1}^{1}+4 S_{1}^{2}
$$

where

$$
\begin{aligned}
S_{1}^{1} & =\sum_{i=1}^{n-1} f_{2 i, 0}^{(k)}+\sum_{j=1}^{m-1} f_{0,2 j}^{(k)}+\sum_{i=1}^{n-1} f_{2 i, 2 m}^{(k)}+\sum_{j=1}^{m-1} f_{2 n, 2 j}^{(k)} \\
& =\sum_{i=1}^{n-1}\left(f_{2 i, 0}^{(k)}+f_{2 i, 2 m}^{(k)}\right)+\sum_{j=1}^{m-1}\left(f_{0,2 j}^{(k)}+f_{2 n, 2 j}^{(k)}\right), \\
S_{1}^{2} & =\sum_{i=1}^{n-1} \sum_{j=1}^{m-1} f_{2 i, 2 j}^{(k)} .
\end{aligned}
$$

The sum in the nodes with one odd index will look like

$$
\sigma_{2}^{(k)}=S_{2}^{1}+2\left(S_{2}^{2}+S_{2}^{3}\right)
$$


where

$$
\begin{aligned}
S_{2}^{1} & =\sum_{i=0}^{n-1} f_{2 i+1,0}^{(k)}+\sum_{j=0}^{m-1} f_{0,2 j+1}^{(k)}+\sum_{i=0}^{n-1} f_{2 i+1,2 m}^{(k)}+\sum_{j=0}^{m-1} f_{2 n, 2 j+1}^{(k)} \\
& =\sum_{i=0}^{n-1}\left(f_{2 i+1,0}^{(k)}+f_{2 i+1,2 m}^{(k)}\right)+\sum_{j+0}^{m-1}\left(f_{0,2 j+1}^{(k)}+f_{2 n, 2 j+1}^{(k)}\right), \\
S_{2}^{2} & =\sum_{i=0}^{n-1} \sum_{j=1}^{m-1} f_{2 i+1,2 j}^{(k)}, \quad S_{2}^{3}=\sum_{i=1}^{n-1} \sum_{j=0}^{m-1} f_{2 i, 2 j+1}^{(k)}
\end{aligned}
$$

correspondingly, and with two odd indices will have a form

$$
\sigma_{3}^{(k)}=\sum_{i=0}^{n-1} \sum_{j=0}^{m-1} f_{2 i+1,2 j+1}^{(k)} .
$$

Thus, the generalized Simpson's cubature formula, with taking into account the method of double recalculation, will have a form (28) and its components will be calculated by formulas (29)-(35). The value $\sigma_{1}^{(k)}$, which is included into the expression (26), only at the zero iteration is calculated by (16) and in other cases it is determined by the branched expression

$$
\sigma_{1}^{(k)}= \begin{cases}2\left[f_{0,2}^{(0)}+f_{2,0}^{(0)}+f_{2,4}^{(0)}+f_{4,2}^{(0)}\right]+4 f_{2,2}^{(0)}, & k=0 \\ \sigma_{4}^{(k-1)}, & k>0 .\end{cases}
$$

The last recurrence relation just provides the calculation process optimal related to the need for computations in double recalculation only for some part of the nodes.

For accuracy posteriori estimation of the calculated double integral, the following inequality is used

$$
\left|I_{k}-I_{k-1}\right|<\varepsilon, \quad k=1,2, \ldots
$$

Example. Calculate the double integral

$$
\iint_{D} \sin \left(\pi^{2} x y\right) d x d y
$$

$D=\{(x, y), 0 \leqslant x, y \leqslant 1\}$ is the unit square.

The integrand is a spatial surface shown in Fig. 2.

The antiderivative of the integrand in (36) can be represented with the help of integral cosine

$$
\begin{aligned}
\operatorname{Ci}(x) & =\int_{\infty}^{x} \frac{\cos (t)}{t} d t=\frac{1}{2}[\operatorname{Ei}[i x]+\operatorname{Ei}[-i x]] \\
& =\ln (\gamma x)+\sum_{n=0}^{\infty}(-1)^{n} \frac{(-1)^{n} x^{2 n}}{(2 n)(2 n) !}
\end{aligned}
$$

i.e. function with a single point of branching [6].

Here $\operatorname{Ei}[z]$ is an integral exponential function

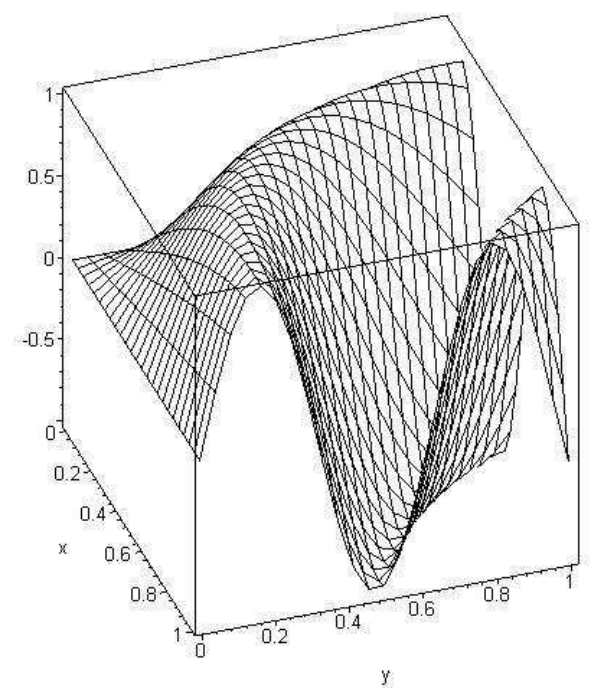

Fig. 2. $f(x, y)=\sin \left(\pi^{2} x y\right)$ function graph.

$$
\operatorname{Ei}(z)=-\int_{z}^{\infty} \frac{e}{t} d t
$$


and $\gamma$ is an Euler's constant, which is determined as follows

$$
\gamma=\lim _{m \rightarrow \infty}\left[\sum_{k=1}^{m} \frac{1}{k}-\ln (m)\right]=0.577215664901533 .
$$

Then the exact value of the integral (36) is calculated by the formula

$$
\begin{aligned}
I_{T} & =\int_{0}^{1} d x \int_{0}^{1} \sin \left(\pi^{2} x y\right) d y \\
& =\pi^{-1}\left(\gamma-\operatorname{Ci}\left(\pi^{2}\right)+2 \ln (\pi)\right) \\
& =0.293900753785 .
\end{aligned}
$$

The calculations were carried out in the system of computer mathematics (SCM) Maple 17 using procedures-functions

$$
\mathrm{Ci}(x)=\gamma+\ln (x)+\int_{0}^{x} \frac{\cos (t)-1}{t} d t
$$

for the system variable Digits $:=24$. Note that the difference in determining (37) of the integral cosine [7] between the built into the core SCM Maple and Mathematica functions (39) is only the representation of improper integrals and the addition of natural $\operatorname{logarithm} \ln x$, leading to various forms of recording with the accuracy to the Euler's constant.

Table 1. Simpson's cubature formula.

\begin{tabular}{|c|c|c|c|}
\hline$k$ & $N=m$ & $I^{(k)}$ & $I_{T}-I^{(k)}$ \\
\hline 0 & 16 & 0.293904016570 & $0.326 \mathrm{E}-5$ \\
\hline 1 & 32 & 0.293900956383 & $0.203 \mathrm{E}-6$ \\
\hline 2 & 64 & 0.293900766427 & $0.126 \mathrm{E}-7$ \\
\hline 3 & 128 & 0.293900754574 & $0.789 \mathrm{E}-9$ \\
\hline 4 & 256 & 0.293900753834 & $0.494 \mathrm{E}-10$ \\
\hline 5 & 512 & 0.293900753788 & $0.308 \mathrm{E}-11$ \\
\hline
\end{tabular}

Table 2. Monte Carlo method.

\begin{tabular}{|c|c|c|}
\hline$N$ & $I_{N}$ & $\left|I_{T}-I_{N}\right|$ \\
\hline 512 & 0.257413264905 & 0.03550 \\
\hline 1024 & 0.263619312307 & 0.03029 \\
\hline 2048 & 0.281496057022 & 0.01251 \\
\hline 4096 & 0.287567672987 & 0.00533 \\
\hline 8192 & 0.287605685862 & 0.00524 \\
\hline 16384 & 0.288771481162 & 0.00513 \\
\hline
\end{tabular}

The results of the developed modifications of the Simpson's cubature formula for different values of partitioning of the integration area are shown in Table 1.

For comparison in Table 2, there are shown the results of calculations of double integral by Monte Carlo method [1] for different values of statistical sampling of the dimension $N$.

The accuracy of Monte Carlo method is determined by the error of generating the pseudorandom sequence of numbers, which are generated on a PC, and the volume of the sample. It can be estimated from the ratio

$$
\delta=\frac{1}{2 \sqrt{N(1-P)}},
$$

where $P$ is the guaranteed probability of error occurrence in the interval $[-\delta, \Delta]$. This error is the value of the order $\underline{O}\left(N^{-1}\right)$ and much greater than in the considered deterministic methods. In addition, for the double integral it is necessary to use two generators of uniformly distributed random numbers in the range $(0,1)$, what, in general, does not provide sufficiently small error and optimal computational costs versus the modified formulas of Simpson with optimal implementation of the principle of double recalculation.

It is necessary to note that for large values of $n, m$, the use of the value of the system variable "by default" (Digits $:=10$ ) leads to the error of rounding-off, which affects not only the loss of accuracy in the eighth significant digit of the mantissa, but it also causes a change in the sign of deviation of the approximate solution from the exact one. 


\title{
3. Conclusions
}

On the basis of the developed software modules and numerical calculations, the benefits of the suggested approach in comparison with the classical schemes are demonstrated in terms of significant reduction of computational costs, in particular, the main memory, processing time, a rounding-off error.

It is necessary to note that used for qualitative comparison Monte Carlo method provides much less accuracy. In terms of the spent CPU time, it can give slightly better results in comparison with the presented formulas, but it will be noticeable only for multiple integrals (starting from triple ones) when selecting a large sample and using a perfect generator of random numbers, connected to the software and hardware complex as physical adapter device.

Being formulated in the paper, the problem of generalization of the method of double and multiple recalculations for the case of multiple integrals can be solved by the application of the above stated algorithm.

[1] Binder K., Heermann D. Monte Carlo Simulation in Statistical Physics. Springer-Verlag, Berlin, Heidelberg (2010).

[2] Gavryliyk I. P., Makarov V. L. Metodi obchislen': pidruchnik u dvokh chast. Kyiv, Vishcha shkola, Ch. 2 (1995), (in Ukrainian).

[3] Veitsblit O. Y. Metod kratnogo pererakhunku. Informatsiyni tekhnologii v osviti. 7, 50-60 (2011), (in Ukrainian).

[4] Lazurchak I. I., Gal' Yu. M. Chislennaya realizatsiya kvadraturnoy formuly Simpsona s avtomaticheskim vyborom shaga. Kyiv, 17, U-89. Dep. v UkrNIINTI (1989), (in Russian).

[5] Lazurchak I. I., Kobil'nik T. P. Sistemi komp'yuternoi matematiki: navchal'niy posibnik. Drohobych, Kolo (2013), (in Ukrainian).

[6] Makarov V.L., Lazurchak I. I. Dvukhstoronniy FD-metod resheniya zadachi Dirikhle dlya uravneniya Gel'mgol'tsa. Differents. uravneniya. 3, n. 35, 388-395 (1999), (in Russian).

[7] Yanke E., Emde F., Lesh F. Special Functions. Nauka, Moscow (1977), (in Russian).

\section{Оптимальні алгоритми реалізації обчислень для кратних інтегралів}

\author{
Бандирський Б. ${ }^{1}$, Гошко Л. ${ }^{1}$, Лазурчак I. ${ }^{2}$, Мельник M. ${ }^{2}$ \\ ${ }^{1}$ Національний університет "Львівсъка політехніка" \\ вул. С. Бандери, 12, 79013, Львів, Украӥна \\ 2 Дрогобицький державний педагогічний університет імені Івана Франка \\ вул. I. Франка, 24, 82100, Дрогобич, Україна
}

\begin{abstract}
Розглянуто оптимальні алгоритми для реалізації кубатурної формули Сімпсона із застосуванням принципу подвійного перерахунку під час обчислення багатократних інтегралів. Порівняно запропонований алгоритм з вбудованими функціями пакета розширень системи комп'ютерної математики на тестовому прикладі обчислення інтегральних тригонометричних функцій. Розширено функціональні можливості використання CKM Mathematica та Maple.
\end{abstract}

Ключові слова: кубатурні формули, оптимізачія обчислень, приниип подвійного перерахунку, інтегральний косинус, системи комп'ютерної математики.

2000 MSC: $60 \mathrm{~J} 10$

Удк: 517.9

Mathematical Modeling and Computing, Vol.4, No.1, pp.1-9 (2017) 